\title{
Supporting Effective User Navigation in Digital Documents
}

Jennifer Pearson

Future Interaction Technology Laboratory, University of Wales, Swansea

csjen@swan.ac.uk

Electronic documents such as PDFs are a large part of the growing digital age and will no doubt form a substantial portion of any paperless office. The harsh truth however, is that electronic documents differ greatly from traditional paper based material. It has been widely documented that the popularity of reading on paper far exceeds the popularity of reading on a computer, with many users choosing to print documents to read them as opposed to reading on a computer screen.

Incorporating tools into digital document readers to aid users in day to day tasks will enhance their performance and hopefully increase user uptake. My research on this topic centres on several areas of document navigation; focusing specifically on current physical (paper) practices in order to enhance their digital equivalents. Each innovative digital tool I have built has then been critically analysed by means of field studies on users from an appropriate target audience. To date, I have investigated three areas of document navigation; Placeholders, Annotation and Indexing, and have several more ideas in mind to take the topic further. I feel that this is a promising digital library topic as it has many practical implications to the community. My work in this area has resulted in three publications and now directional feedback would be most welcome to finish the final stages of my $\mathrm{PhD}$. 